\title{
Analysis of diagnosis and treatment of aural vertigo
}

\author{
Xiaohui Zhang, Xiaoping Sun, Zhaoxia Xu* Aiping Guo \\ Department of Otolaryngology, The Third Affiliated Hospital of Inner Mongolia Medical University, China
}

Received: January 23, 2017

DOI: $10.14725 /$ dcc.v4n1p17

\author{
Accepted: February 28, $2017 \quad$ Online Published: March 10, 2017 \\ URL: http://dx.doi.org/10.14725/dcc.v4n1p17
}

\begin{abstract}
A case of aural vertigo in the Third Affiliated Hospital of Inner Mongolia Medical University was collected. The diagnosis and treatment of the disease were analyzed by experts, and a variety of causes of the disease were discussed, in order to achieve personalized treatment and satisfactory curative effect.
\end{abstract}

Key Words: Aural vertigo, Diagnosis and treatment

\section{Medical record}

\subsection{General information}

A 51-year-old female patient was admitted to our hospital due to vertigo accompanied by a stuffy right ear and hearing loss for duration of 7 days. Prior to admission, the patient suddenly suffered from vertigo accompanied by a stuffy sensation of the right ear, visual rotation, nausea, vomit, and dared not turn the neck. Vertigo could be induced whenever turning neck, and dizziness lasted about 1 minute each time. In the community clinic, the patient was treated with Ginkgo Leaf Extract and Dipyridamole Injection ( $20 \mathrm{ml} /$ day) by intravenous drip for 7 days, and she felt much better. Vertigo appeared again while changing the position of the neck. In that case, the patient decided to see our doctors today. Past medical history included Meniere's disease for three years during which broke out twice. The pure tone audiometry examination was performed again when the patient felt better, only ear low-frequency hearing threshold $(250 \mathrm{~Hz}, 30 \mathrm{~dB}$, $500 \mathrm{~Hz}, \mathrm{~dB} 25)$ mildly decreased. That diabetes and hypertension coronary heart disease, other chronic disease, and ototoxic medication history were all denied.

\subsection{Physical examination}

Data on physical examination included $\mathrm{T} 36.5^{\circ} \mathrm{C}, \mathrm{P} 80 / \mathrm{min}$, R 19/min, BP 120/70 mmHg. The external auditory canal was unobstructed, the tympanic membrane was intact and the sign was clear. Neck soft, and no abnormalities were found in heart, lung and abdomen.

\subsection{Auxiliary examination}

Pure tone audiometry presented flat curve, indicating moderate sensorineural hearing loss in the right ear and speech frequency average hearing threshold of $70 \mathrm{~dB}$. The left ear hearing threshold was normal. The acoustic guidance showed bilateral "A" tympanum patterns, and the left side of the acoustic reflex was ipsilateral and contralateral. Transient otoacoustic emissions confirmed the normal condition of the right ear, and the abnormalities of the left ear. The waveform was not shown during electrocochleogram examination in the right ear. The auditory brainstem response showed a marked prolongation I wave latency in the right ear and normal in the left ear. The latency difference of binaural $\mathrm{V}$ wave was less than $0.3 \mathrm{~ms}$. The patient sitting on the bed, headed to the side of $45^{\circ}$, lay down quickly with head drooping out of bed to the side of $30^{\circ}$, presented vertical torsional nystagmus; A few seconds after the patient was brought to a sitting position, reversal of nystagmus appeared. Tube stone disease duration was $<1 \mathrm{~min}$ and ridge cap stone disease duration was $\geq 1$ min. Nystagmus was induced on any side, the pathological changes could be

\footnotetext{
*Correspondence: Zhaoxia Xu; E-mail: Xuzhaoxia@163.com; Address: Department of Otolaryngology, The Third Affiliated Hospital of Inner Mongolia Medical University, China.
} 
tracked by PC-BPPV examination. Dix-Hallpike check suggested right side positive. There was no abnormality in CT of temporal bone.

\subsection{Primary diagnosis}

Vertigo of unknown origin:

(1) Benign paroxysmal positional vertigo

(2) Sudden deafness (right)

(3) Meniere's disease?

\subsection{Diagnosis and treatment}

After admission, the modified Epley maneuver was performed (the patient was seated and the head was first turned to the affected side for $45^{\circ}$, then lying down rapidly, and the head was lower than the shoulder. Then, after turning to the contralateral side for $45^{\circ}$, the patient turned to the contralateral side for $90^{\circ}$ of four directions to face up. Each direction would last for 1 minute. If vertigo occurred, side rotation was stopped, and then continued to side turn after the disappearance of nystagmus for $30 \mathrm{~s}$ ). Because of the poor effect of the first reduction, the patient had no improvement in vertigo, the modified Semont and modified Epley technique were used. The modified Semont Canalith relief method was introduced as follows: sitting position, head to the side to rotate $45^{\circ}$ to its rapid horizontal to the side, observed the symptom of nystagmus. Maintained the head position for 2-3 min after nystagmus disappeared, and then helped the patient sit up quickly and immediately turned to the side to lay down, and kept head down to rotate $45^{\circ}$ to observe the nystagmus. 5 min later, changed into supine position and helped the patient slowly sit straight to make the stone into the utricle. After the reset, the patient felt vertigo significantly improved. Ginkgo Leaf Extract and Dipyridamole Injection ( $20 \mathrm{ml} /$ time, 1 time/day), ATP Injection (20 mg/time, 1 time/day), Coenzyme A Injection (100 U/ time, 1 time/day), Mecobalamin Injection (500 $\mu \mathrm{g} /$ time, 1 time/day intravenous injection for 10 days), Dexamethasone Injection (10 mg/day, 1 time/day), after potted for three days, changed the dose (5 mg/day, 1 times/day) and potted for another three days. Flunarizine Hydrochloride Capsules ( $5 \mathrm{mg} /$ time, 1 time/day), orally. Hyperbaric oxygen therapy, right ear physiotherapy, 1 time/day. Ten days after the treatment, the patient felt better in the right ear hearing. Before the treatment, pure tone audiometry showed language frequency threshold $70 \mathrm{~dB}$ (see Figure 1), cochlear electrogram was not elicited (see Figure 2), after treatment, the review of pure tone audiometry showed language frequency threshold recovery by $20 \mathrm{~dB}$ (see Figure 3), electrocochleogram figure generated and AP latency extended (see Figure 4).

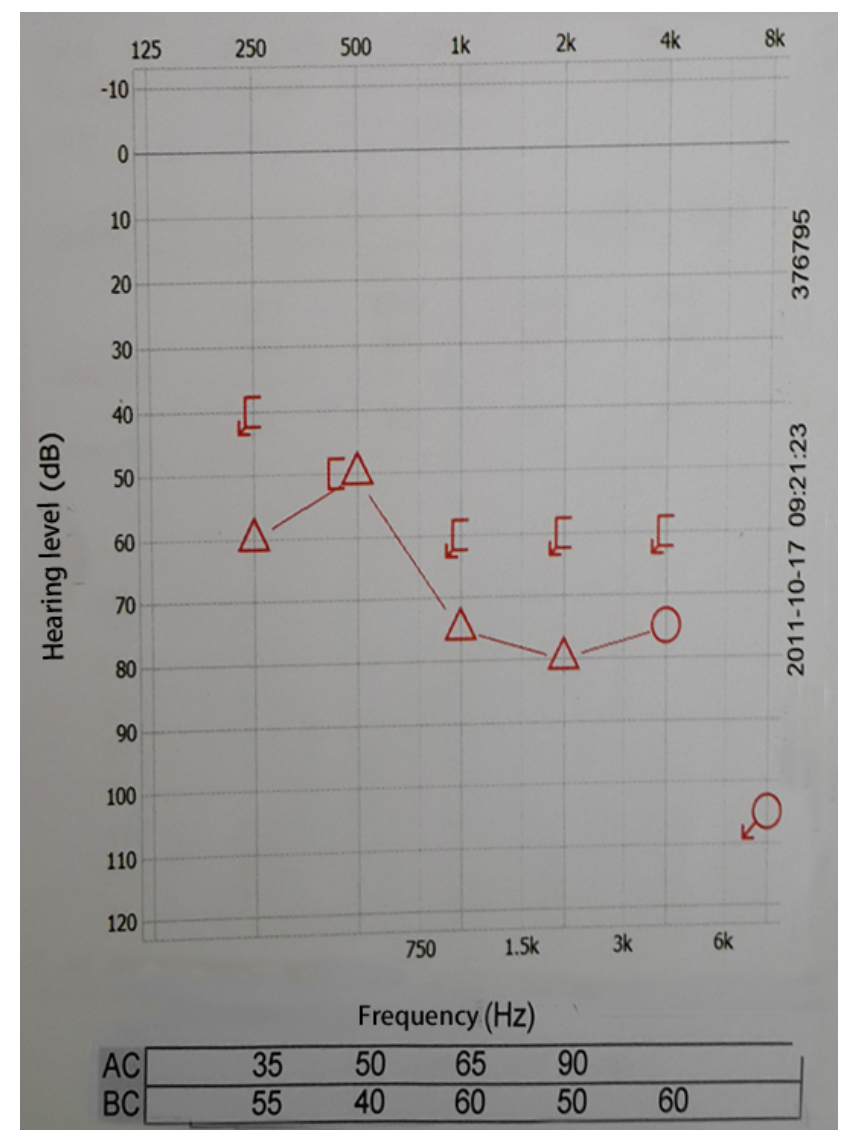

Figure 1: Pure tone audiometry before treatment

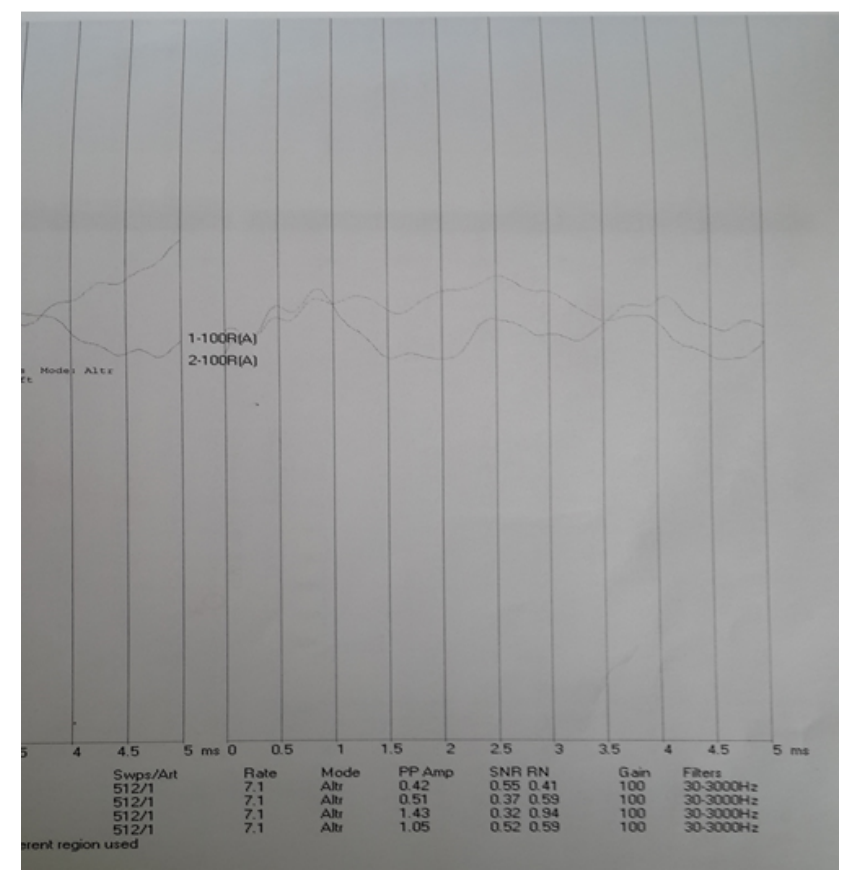

Figure 2: Cochlear electrogram before treatment 


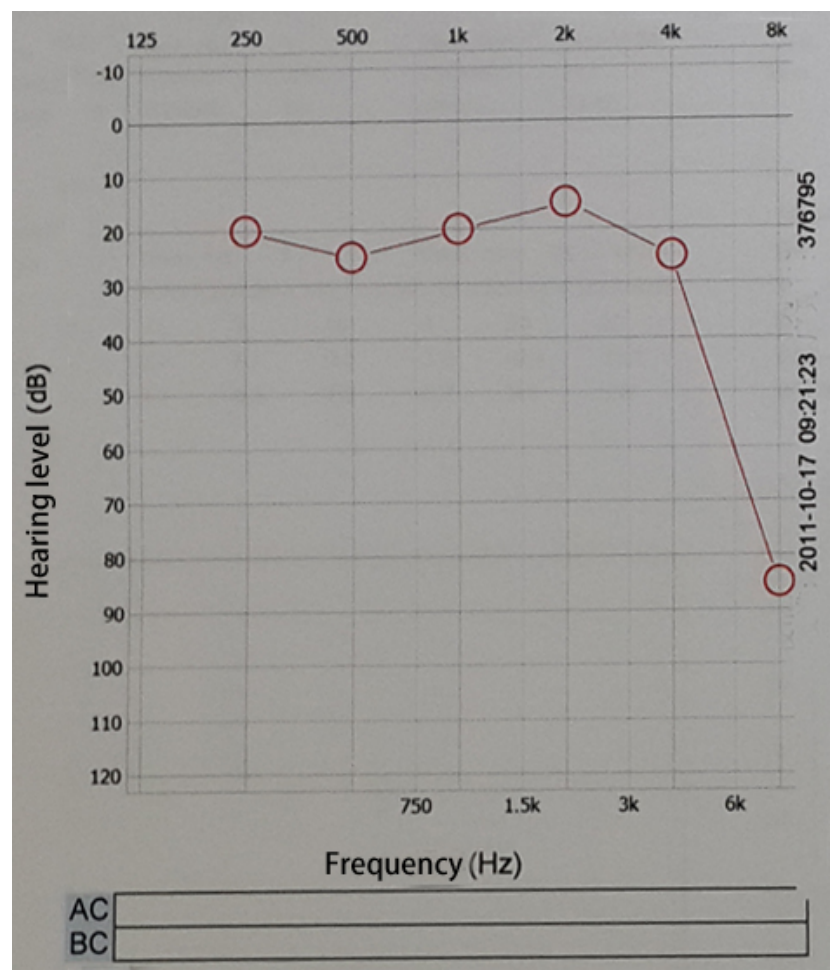

Figure 3: Pure tone audiometry after treatment

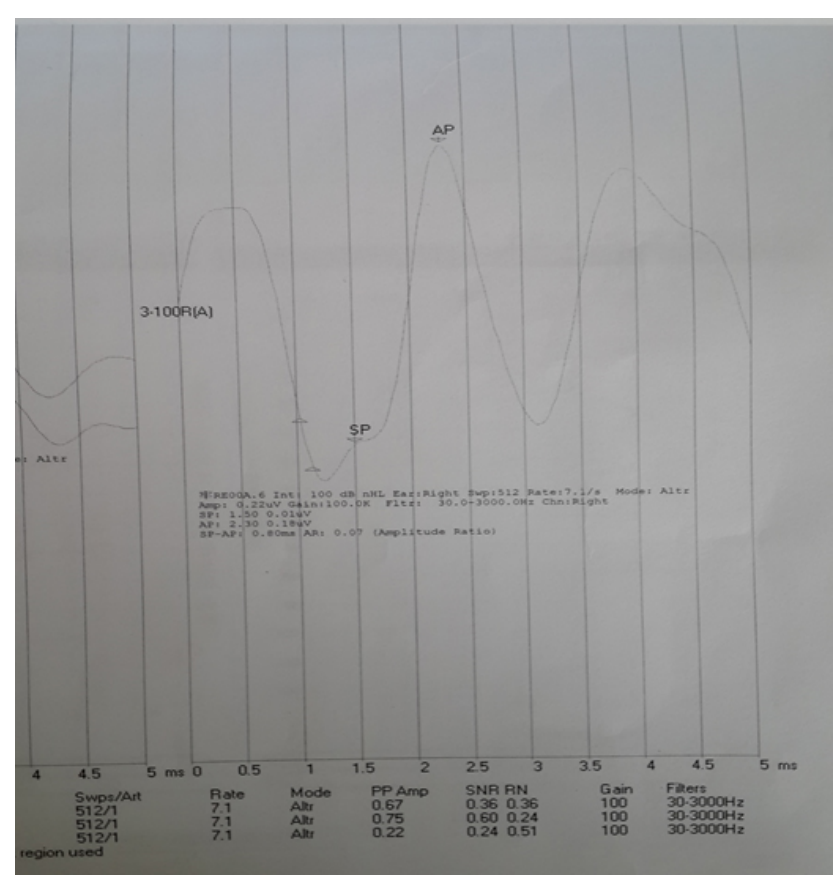

Figure 4: Cochlear electrogram after treatment

\section{Discussion}

\subsection{Dr. Sunxiao Ping}

Dr. Sunxiao Ping is the director of Department of Otolaryngology at the Third Affiliated Hospital of Inner Mongolia

Published by New Century Science Press
Medical University, specializing in diagnosis and treatment of ear diseases.

The concept of benign paroxysmal positional vertigo was first proposed by Barany in 1921. It is a transient vertigo induced by a head movement to a specific location. The disease is a vertigo seizure caused by the degeneration of the otolith or the detachment of the otolith particles into the semicircular canal when the body position changes. The key to BPPV treatment is to allow the otolith on the semicircular canal or crest cap to leave the semicircular canal and enter the vestibule to be reabsorbed. The cause of the disease is not clear. It is generally believed that the thinning of the macula caused by: vertebrobasilar insufficiency leads to the falling of the otolith into the semicircular canal or ampullary crest; the pathological changes of the middle ear and inner ear; the aging of the otolith membrane and the loss of the otoliths. The incidence of this disease accounted for about $1 / 3$ of the patients with vertigo, with self-limiting peripheral vestibular disease, and was often misdiagnosed as cervical disease, Meynier disease, vestibular neuronitis and other diseases by which examination and a large number of drug treatment were performed, but failed to achieve satisfactory curative effect. In 1952, Dix and Hallpike described the characteristics of BPPV and the technique of body position experiment, and diagnosed the disease by observing the position change of nystagmus. The treatment effect was better. The diagnosis and treatment of BPPV was estimated by observing the nystagmus. ${ }^{[1]}$ In the past, $30 \%$ of the nystagmus was ignored by naked eye observation, and the nystagmus and fixation nystagmus were not easily distinguished, which reduced the diagnostic rate of the disease. At present, the whole process of body position test and reposition can be carried out under video surveillance by video nystagmus, which is very important to observe the direction and characteristics of nystagmus, and to eliminate the fixation and increase the detection of nystagmus. It is crucial to the diagnosis of BPPV and the side of the disease. The patient was repositioned successfully twice, the vertigo was improved and positional nystagmus disappeared completely. Wu Ziming et al. ${ }^{[2]}$ reported that abnormal otolith displacement during otolith repositioning was not uncommon. The incidence of abnormal otolith displacement was $3.3 \%$, Foster et al. ${ }^{[3]}$ indicated that the incidence rate was $16 \%$. In the course of reduction, if the patient failed to perform position change according to the doctor's instructions or the position change was not in place, the otolith would fall into the other half of the tube. The key point of successful reduction is the correct judgement of the shedding of the otolith. ${ }^{[4]}$ Therefore, it is necessary to observe the changes of nystagmus carefully under the video nystagmus in the process of resetting, so as to accurately diagnose the displacement of the otolith. If the reset process appears inconsistent with the expected nystagmus, such as vertical torsional nystagmus into horizontal nystagmus, or the process does not relieve or aggravate the symptoms, abnormal otolith shift into adjacent 
semicircular canal should be taken into consideration. Vertical torsional nystagmus of the patient was $\geq 1$ min during Dix-Hallpike examination so that the diagnosis of crest cap stone disease was made. Using Semont tube stone relief with modified Epley maneuver, the stones successfully entered the vestibular pool, so the symptoms of vertigo were improved.

\subsection{Dr. Zhaoxia Xu}

Dr. Zhaoxia Xu is the deputy director of Department of Otolaryngology at the Third Affiliated Hospital of Inner Mongolia Medical University, specializing in diagnosis and treatment of ear diseases.

Sudden deafness refers to sudden, unknown sensorineural hearing loss, most of which are unilateral deafness with no more aura, while a small number of patients accompanied by mild cold, fatigue or emotional history before the onset of the disease. Hearing loss occurs suddenly, and the patient's hearing drops to severe level in a few minutes or hours. A few patients may reach profound level within 3 days. The main clinical manifestations are unilateral hearing loss, accompanied by tinnitus, ear congestion, dizziness, nausea, vomiting, etc. The etiology of sudden deafness is unknown. At present, theories such as virus infection, circulatory disorder, autoimmune and membranous labyrinth rupture are the main aspect. It is generally believed that the inner ear blood supply disorder is the main cause of sudden deafness, ${ }^{[5]}$ the degree of hearing loss is related to the blood flow velocity, blood flow and cross sectional area of the cochlea. The blood supply of the inner ear comes from the labyrinthine artery. The physiological activity of inner ear hair cells needs high oxygen consumption, with poor tolerance to hypoxia, decreased blood pressure or partial pressure of circulating carbon dioxide may cause the decrease of cochlear lymphatic oxygen tension, thereby reducing the amount of oxygen supply in the inner ear. Therefore, cochlear microcirculatory dysfunction caused by various causes can result in hearing organ dysfunction and hearing loss. Audiological examination: pure tone audiometry showed hearing curve suggestive of severe sensorineural hearing loss in a high frequency descent type. Acoustic impedance test: normal tympanic pressure curve. Otoacoustic emissions and cochlear findings indicated cochlear damage. Vestibular function tests are usually performed during the remission stage of vertigo, and the common cold and hot alternate test combined with nystagmus recording can be normal, decrease or disappear completely. Imaging examination: temporal bone $\mathrm{CT}$, internal auditory canal MR indicated that the internal auditory canal and the brain were without obvious organic disease. The patient experienced the disease by sudden in a short period of time, and the right ear hearing decreased to the profound level. While, the average hearing threshold of language frequency was $70 \mathrm{~dB}$, acoustic immittance showed normal tympanic cavity pressure curve. The transient otoacoustic emission showed abnormal, and the cochlea did not elicit. There was no abnormality in CT of temporal bone. So, the diagnosis of sudden deafness in right ear was made.

\subsection{Dr. Aiping Guo}

Dr. Aiping Guo is the deputy director of Department of Otolaryngology at the Third Affiliated Hospital of Inner Mongolia Medical University, specializing in diagnosis and treatment of ear diseases.

Meniere's disease is a endolymphatic hydrops caused by circulatory disorders of the inner ear, resulting in repeated episodes of rotatory vertigo, fluctuating hearing loss, tinnitus, and ear fullness. It includes cochlear symptoms and disorders of vestibular function. When patients have Meynier's disease, the lymphatics in the cochlear duct will increase, making the vestibular membrane toward the vestibular step expansion, while the basement membrane moves toward the scala tympani. These are the structural changes caused by the mechanical pressure induced by the formation of the lymph fluid, as well as the main factor of hearing loss in this disease. ${ }^{[6]}$ It is inevitable to separate the auditory hair cells attached to the tectorial membrane, and lose the movement of scissors with the fluctuation of the lymph. Therefore, the early stage of endolymphatic hydrops is mainly caused by the partial displacement of the basilar membrane of the longer fibers of the cochlea, which is clinically characterized by low frequency hearing impairment.

With the increasing pressure of lymph fluid to the surrounding structure of the cochlea, the most common cause of rupture is the vestibular membrane. Any part of the membrane labyrinth may rupture and twist, forming a large amount of potassium ion in the endolymph and entering the lymph and Corti lymph, causing the toxication of the auditory hair cells. The hair cell can not convert the fluid produced by sound into electrical signal, which can be displayed in the change of action potential (-SP/AP > 0.4). There may be a healing of membranous structures between the two ruptures. Therefore, recurrent vertigo and fluctuating hearing loss may be the clinical manifestation of this fracture healing cycle. In the development stage of Meniere's disease, sensory organs exist persistent morphological changes. Changes in Corti's apparatus include loss of hair cells, atrophy of supporting cells, deformation and atrophy of the tectorial membrane, and vestibular membrane involvement in the Corti apparatus. When the potassium contaminated lymph fluid reaches the apex of the scala tympani through the cochlear foramen, it may change the electrolyte environment of the peripheral nerve fibers in this region, resulting in atrophy of the cells. Hence, repeated attacks of the disease can completely cause damage to hair cells and even cochlear nerve roots, leaving permanent hearing impairment. The 
patient with past medical history was checked with ear lowfrequency hearing threshold decreased slightly $(250 \mathrm{~Hz}, 30$ $\mathrm{dB}, 500 \mathrm{~Hz}, 25 \mathrm{~dB}$ ) after pure tone audiometry twice since the onset of the disease. It can be concluded that the patient was in the early stage of endolymphatic hydrops, that is, only the physical change of basilar membrane displacement occurred. In addition to hearing loss accompanied by low frequency hearing curve in the patient, cochlear electrogram could not show the abnormalities, which does not conform to the hearing characteristics of Meniere's disease. The incidence of Meniere's disease onset can be denied.

\subsection{Dr. Xiaohui Zhang}

Dr. Xiaohui Zhang is the deputy director of Department of Otolaryngology at the Third Affiliated Hospital of Inner Mongolia Medical University, specializing in diagnosis and treatment of ear diseases.

Vertigo is a sense of self produced by the spatial orientation and balance dysfunction of the body, which presents as a kind of motion illusion. Vestibular peripheral vertigo appears a sense of rotation or dumping, accompanied by paroxysmal nystagmus, imbalance (deflecting, standing instability or dumping) and vegetative nerve symptoms (pale, nausea, sweating, blood pressure and pulse rate change), which is generally shared by nystagmus and vestibular. In addition to vertigo, nystagmus and vestibular function, tinnitus is accompanied by tinnitus and hearing loss, and most of them are unilateral. No other signs of nervous system. Benign paroxysmal positional vertigo, Meniere's disease, and sudden deafness are the most common diseases causing aural vertigo.

The treatment of aural vertigo must first clarify the cause of the disease so as to carry out individualized treatment. The patient had a short history of clear hearing loss, and hearing test in pure tone audiometry indicated right moderate sensorineural hearing loss, flat curve, the linguistic thresholds was $70 \mathrm{~dB}$. The auditory brainstem response showed prolongation of the right ear I wave latency. Cochlear electrogram was not elicited. It was suggested that cochlear lesions were present in the patient. So right ear sudden deafness can be diagnosed clearly. The inner ear blood supply disorder is the main cause of sudden deafness. The blood supply from the inner ear mainly comes from the labyrinthine artery. The labyrinthine artery enters the inner ear through the inner ear canal and divides into the vestibular artery and the common cochlear artery. The branches of the vestibular

\section{References}

[1] Cui ZH. The diagnosis and treatment of Meniere's disease status. Practical Journal of Medicine. 2006; 23(7): 866-868. artery supply the utricle and the upper and lateral parts of the saccule, and part of the superior semicircular canal and the outer semicircular canal. The common cochlear artery is divided into the vestibular cochlear artery and the cochlear proper artery. The former branches supply the lower and medial portions of the oval and the utricle and the saccule, and the posterior semicircular canal and the total foot and the base of the cochlea. The latter also refers to spiral artery, which mainly distributes at the circle and the top ring to the cochlea. In that case, cochlear lesions often coexist with vestibular symptoms. The main treatment is to promote blood circulation to remove blood stasis, improve the circulation of the inner ear, nutrition nerve and hormone and reduce nerve edema, supplemented by hyperbaric oxygen and local physical therapy to get a satisfactory effect. On the one hand, the hyperbaric oxygen can improve the partial pressure of oxygen and the hypoxia state of the inner ear auditory organs, prevent the capillary degeneration of the inner ear and cochlear nucleus, promote the proliferation of hair blood tubule, establish collateral circulation, and promote the functional recovery of auditory cells. On the other hand, hyperbaric oxygen is able to improve the local hypoxia state, make local capillary contraction, reduce capillary permeability to alleviate the exudation and ease the effect of local edema. ${ }^{[7]}$ Besides, the hyperbaric oxygen can be used to reduce hematocrit, improve erythrocyte elasticity and hamper platelet aggregation, and reduce blood viscosity. It also lightens leukocyte adhesion to endothelial cell, lowers blood viscosity, reduces leukocyte aggregation, improves microcirculation and blood perfusion of injury. ${ }^{[8]}$ In addition to the improvement of microcirculation, hyperbaric oxygen can also promote the absorption and utilization of drugs, so as to achieve the complementary effect. ${ }^{[9]}$ In addition, because of the presence of positive Dix-Hallpike findings in this patient, benign paroxysmal positional vertigo can confirmedly be diagnosed. As for benign paroxysmal positional vertigo, the semicircular canal where otolith located off could be confirmed according to position experiment and electronystagram examination. So we could take the corresponding manipulative reduction, and the patient felt better. Therefore, the vertigo of this patient is caused by two factors, so it is necessary to make a comprehensive judgment in the treatment, and take the corresponding treatment strategy to achieve satisfactory curative effect.

\section{Conflicts of Interest Disclosure}

The authors have no conflicts of interest related to this article.

[2] Wu ZM, Zhang SZ, Liu XJ. The shift of otolith in repositioning of benign paroxysmal positional vertigo. Chinese Journal of Otorhinolaryngology Head and Neck Surgery. 2009; 44(8): 623-626. PMid: 19961767.

[3] Foster CA, Zaccaro K, Strong D. Canal conversion and reentry: 
a risk of Dix-Hallpike during canalith repositioning procedures. Otol Neurotol. 2012; 33(2): 199-203. PMid: 22143303. https: //doi.org/10.1097/MA0.0b013e31823e274a

[4] Titus SI, Rogers C. Clinical evaluation of posterior canal benign paroxysmal positional vertigo. Niger Med J. 2012; 53(2): 94-101. PMid: 23271854. https://doi.org/10.4103/0300-1652. 103550

[5] Kanzaki J, Inoue Y, Ogawa K, et al. Effect of single-drug treatment on idiopathic sudden sensorineural hearing loss. Auris Nasus Larynx. 2003; 30(2): 123-127. https://doi.org/10.1016/ S0385-8146(03) 00009-9
[6] Shi HL, Wang GB. Research progress of Meniere's disease in inner ear lymph magnetic resonance imaging. Chinese Interventional Imaging. 2013; 10(3): 187-190.

[7] Yuan ZL. Clinical analysis of hyperbaric oxygen therapy for sudden deafness. Chinese Medical Journal. 2009; 6(9): 251.

[8] Huang H, Li N, Gu J, et al. Comparative study of hyperbaric oxygen therapy and drug therapy for sudden deafness. Chongqing Medical Sciences. 2010; 39(8): 938.

[9] Guo H, Liu QH, Xiao P. Research progress of hyperbaric oxygen therapy on cerebral infarction J. Clinical Medical Research. 2004; 21(6): 584-586. 